\title{
Efecto del tratamiento térmico en la estabilidad de geles obtenidos a partir de harinas de quinua
}

\author{
Yesika B. Lopez, Diego F. Roa y Jesús E. Bravo \\ Departamento de Ingeniería Agroindustrial, Universidad del Cauca, Popayán; Calle 5 No. 4 - 70, Popayán - 190003, \\ Colombia. (Correo-e: yesikalopez@unicauca.edu.co; jebravo@unicauca.edu.co; *droa@unicauca.edu.co)
}

Recibido Jun. 24, 2021; Aceptado Ago. 23, 2021; Versión final Sep. 20, 2021, Publicado Feb. 2022

\begin{abstract}
Resumen
El objetivo del presente estudio fue determinar el efecto del tratamiento térmico sobre las estructuras secundarias de las proteínas y sobre la estructura interna del almidón de harina de quinua. Se empleó grano de quinua previamente seleccionado y tamizado de la variedad Tunkahuan. Se determinaron los cambios en las proteínas y el almidón en diferentes geles. Los resultados mostraron diferencias significativas en las bandas $\beta$-sheet $\left(1627 \mathrm{~cm}^{-1}\right), \beta$-turns $\left(1661 \mathrm{~cm}^{-1}\right)$ y $\alpha$-helix $\left(1653 \mathrm{~cm}^{-1}\right)$. El almidón ubicado en la superficie del perisperma fue más amorfo. Los geles formados a partir de las harinas del germen fueron menos firmes que los geles formados a partir de grano pulido. Se concluye que la molienda abrasiva resulto ser una operación eficiente para obtener dos tipos de harinas a partir del grano de quinua, una harina con un contenido de proteína y grasa elevado (harina del germen) y una harina con alto contenido de almidón (harina del perisperma).
\end{abstract}

Palabras clave: tratamiento térmico; estabilidad; gel; estructuras secundarias; proteína; cristalina; amorfa; retrogradación

\section{Effect of heat treatment on the stability of gels obtained from quinoa flours}

\begin{abstract}
The primary objective of the present study was to determine the effect of heat treatment on quinoa flour protein secondary structures and internal starch structure. Quinoa grains of the Tunkahuan variety were selected and protein and starch changes in different gels were determined. The results showed significant differences in $\beta$ sheet $\left(1627 \mathrm{~cm}^{-1}\right), \beta$-turns $\left(1661 \mathrm{~cm}^{-1}\right)$, and $\alpha$-helix $(1653 \mathrm{~cm}-1)$. The starch located on the surface of the perisperm was amorphous. Germ flour gels were less solid than polished grain gels. It is concluded that abrasive grinding was an efficient procedure to obtain two types of flour from quinoa grains, one with high protein and fat content (germ flour) and another with high starch content (perisperm flour).
\end{abstract}

Keywords: heat treatment; stability; gel; secondary structures; protein; crystalline; amorphous; retrogression 


\section{INTRODUCCION}

La quinua (Chenopodium Quinoa Willd) es un pseudocereal cultivado principalmente en la zona andina desde hace 7.000 años y su semilla ha sido reconocida como un súper alimento debido a su contenido de proteínas, su digestibilidad y su composición equilibrada en aminoácidos esenciales (Dussán-Sarria et al., 2019). Los estudios sobre la quinua se han centrado en el mejoramiento genético y la adaptación de cultivares a diferentes condiciones agronómicas (Morillo et al., 2017); desde el aprovechamiento del grano como alimentos, las investigaciones han abarcado la caracterización fisicoquímica y nutricional encontrando que la proteína de la quinua tiene un perfil de aminoácidos equilibrado (Le et al., 2021) y de alta digestibilidad proteica (Ghumman et al., 2021); su almidón es poliformico, con entalpia de gelatinización entre 7.18 a $10.34 \mathrm{~J} / \mathrm{g}$ (Xing et al., 2021), con altos niveles de amilopectina; el contenido de grasa oscila entre $3.9 \mathrm{~g}$ a $5.2 \mathrm{~g}$ según la variedad (Rodríguez et al., 2021).

En cuanto a operación agroindustriales, la molienda húmeda de pseudocereales ha sido estudiada por algunos autores (Calzetta et al., 2006; Ballester-Sánchez et al., 2020), los resultados mostraron que las condiciones de humectación afectan directamente las propiedades fisicoquímicas y tecnofuncionales del almidón y de la proteína, lo que posiblemente incrementa el uso de estos como materia prima en la elaboración de productos alimenticios. Sin embargo, la tecnología de molienda en húmedo es costosa en comparación a la molienda seca, además genera efluentes que obliga a la industria a tener un sistema de tratamientos, lo cual, resulta en algunas ocasiones difíciles de implementar en pequeñas industrias (Ngamnikom y Songsermpong, 2011). Es por esta razón, que Roa et al., $(2017,2020)$ desarrollaron un método de molienda abrasiva por medio del cual lograron obtener harinas de amaranto y quinua con diferente composición química; las cuales fueron caracterizadas según las propiedades físicas y reológicas. La literatura indica, que la inclusión de harina de quinua incrementa los valores de G' y G", además de aumentar la elasticidad y la mejora de la estructura (Turkut et al., 2016).

La capacidad gelificante de las harinas, está influenciada por la competencia física por el agua entre la gelificación de proteínas y la gelatinización del almidón, y también, se relaciona con sus diferentes tamaños de partícula y contenido de proteínas, carbohidratos y lípidos, y las interacciones entre ellos (Solaesa et al., 2020). Estudios anteriores (Ahmed et al., 2018) indican que las fracciones de tamaño medio presentan estructuras de gel más débiles mientras que fracciones más gruesas conducen a geles más consistentes, con los módulos elásticos (G') y viscosos (G"). Por lo tanto, en este trabajo se estudió el efecto térmico sobre las propiedades estructurales y reológicas de los geles de las harinas de quinua obtenidas a diferentes condiciones de molienda abrasivas.

\section{METODOLOGÍA}

La metodología se presenta en subsecciones: en una se describe la obtención del grano de quinua, en la segunda se describen los procesos tecnológicos aplicados al grano de quinua y sus harinas y en la tercera subsección se detallan los métodos para determinar las propiedades estructurales y reológicas.

\section{Materia prima}

Se empleó grano de quinua previamente seleccionado y tamizado de la variedad Tunkahuan, del Distrito de Los Milagros Bolívar, Cauca en Colombia. El grano fue proporcionado por la empresa SEGALCO S.A.S.

\section{Obtención de harina de quinua por molienda abrasiva}

El grano de Quinua se sometió a un proceso de molienda mediante un molino experimental de arroz (GranEL, modelo C-100, Bogotá, Colombia), el cual tiene una cámara de abrasión para obtener harinas de quinua con diferentes grados de molienda. Se utilizaron $88 \mathrm{~g}$ de grano entero con el fin de mantener la eficiencia de la cámara de pulido. El tiempo de molienda empleado fue de 10 y 40 minutos obteniendo fracciones pulidas en forma de harina, las cuales se denominaron FP-10 y FP-40. Las harinas del grano pulido y del grano entero se obtuvieron mediante un proceso de molienda tradicional, denominadas C-10, C-40 y C1, respectivamente. Mediante el tiempo y la cantidad de grano en la cámara de pulimiento se realizó según la metodología establecida por Roa et al., (2020). Las harinas fueron pasadas por un tamiz con una abertura de $125 \mu \mathrm{m}$ y otras de $250 \mu \mathrm{m}$.

\section{Tratamiento térmico}

El tratamiento térmico se realizó mediante la metodología establecida por Bolade et al., (2002) con algunas modificaciones. Se prepararon dispersiones de harina en agua con una relación (1:3), posteriormente las dispersiones se homogenizaron durante 2 minutos a $9000 \mathrm{rpm}$ en un equipo ultraturrax, luego las dispersiones fueron calentadas a $90^{\circ} \mathrm{C}$ durante 40 minutos empleando el termostato marca polystat, posteriormente las 
muestras se secaron a $50^{\circ} \mathrm{C}$ durante 16 horas en un horno de convección forzada, y finalmente las harinas obtenidas se almacenaron a $5{ }^{\circ} \mathrm{C}$, en bolsas de polietileno para evitar transferencia de agua al material.

\section{Espectroscopía infrarroja de rango medio por ATR}

Los espectros de FTIR se registraron en un espectrómetro IRAFFINITY-1S (Shimadzu Corp., Kyoto, Japón) equipado con el software LabSolutions IR (Shimadzu Corp., Kyoto, Japón). El espectro fue adquirido utilizando el modo de reflexión entre $600 \mathrm{~cm}^{-1}$ y $4000 \mathrm{~cm}^{-1}$. Cada sistema se midió por triplicado a $25^{\circ} \mathrm{C}$. El análisis espectral se realizó utilizando OriginPro versión 8. Los espectros fueron corregidos y normalizados (entre 0 y 1) relacionando el pico de transmitancia más alto para la presentación de las figuras. Posteriormente, a los espectros se les aplicó un proceso de deconvolución según el protocolo establecido por Yang et al., (2015) con el fin de obtener información detallada para determinar los cambios en la estructura secundaria de las proteínas, entre las bandas a $1600 \mathrm{~cm}^{-1}$ a $1700 \mathrm{~cm}^{-1}$, así como, en la conformación estructural del almidón se empleó el protocolo establecido por Roa et al., (2020) entre las bandas a $875 \mathrm{~cm}^{-1}$ para $1175 \mathrm{~cm}^{-1}$. A los espectros se les determinó el área bajo la curva de los picos de interés por medio de la integración de los picos. La asignación de las bandas se realizó de acuerdo con la tabla 1.

Tabla 1: Estructura secundaria de las proteínas (Datos tomados de Yang et al., 2015)

\begin{tabular}{|c|c|c|c|}
\hline Estructura secundaria & $\mathrm{cm}^{-1}$ & Estructura secundaria & $\mathrm{cm}^{-1}$ \\
\hline$\beta$-sheet & $1619 \pm 2$ & 310-helix & $1661 \pm 3$ \\
\hline$\beta$-sheet & $1627 \pm 2$ & $\beta$-turns & $1669 \pm 2$ \\
\hline$\beta$-sheet & $1635 \pm 2$ & $\beta$-turns & $1675 \pm 3$ \\
\hline$\beta$-sheet & $1641 \pm 3$ & $\beta$-turns & $1684 \pm 2$ \\
\hline Random coil & $1646 \pm 2$ & $\beta$-sheet & $1689 \pm 2$ \\
\hline$\alpha$-helix & $1653 \pm 2$ & $\beta$-sheet & $1695 \pm 2$ \\
\hline
\end{tabular}

\section{Comportamiento viscoelástico}

Las propiedades viscoelásticas de las muestras se determinaron según lo descrito por Zhu y Wang (2012), con algunas modificaciones y se analizaron utilizando un reómetro (TA INSTRUMENTS, AR 1500, New Castel, EE. UU.) con un sistema de placa Peltier con control de temperatura y una geometría de placa paralela lisa de $40 \mathrm{~mm}$ de diámetro. Se cargaron aproximadamente $2 \mathrm{~g}$ de dispersión sobre la placa inferior y la placa superior se bajó a un espacio de $1 \mathrm{~mm}$. Se limpió el exceso de dispersión y se eliminó del borde exterior de la geometría de la placa superior. Luego, se añadió aceite de parafina al borde exterior de la dispersión para evitar la evaporación de agua durante la medición. La velocidad de calentamiento fue de $10{ }^{\circ} \mathrm{C} / \mathrm{min}$ a $1 \mathrm{~Hz}$ y $0,01 \%$ de deformación. Los módulos elásticos y viscosos ( $G^{\prime}$ y $\left.G^{\prime \prime}\right)$ fueron determinados, respectivamente.

\section{Estabilidad de gel}

Las harinas se sometieron a un proceso de gelificación en un exceso de agua empleando una relación (3:1) a $90^{\circ} \mathrm{C}$ durante 40 minutos utilizando el termostato marca polystat, una vez formado el gel se almacenaron a $5^{\circ} \mathrm{C}$ por 24 horas. Las propiedades viscoelásticas de los geles se analizaron según lo descrito por Zhu y Wang (2012), con algunas modificaciones y se midieron en un Reómetro (TA INSTRUMENTS, AR 1500, New Castel, EE. UU.) Utilizando geometría de placas paralelas en modo de rotación con tensión controlada. Se realizó un barrido de amplitud ( $1 \%$ a $100 \%$ ) a $25^{\circ} \mathrm{C}$ y frecuencia angular de $5 \mathrm{~Hz}$, para establecer la región viscoelástica lineal (LVR). Los barridos de frecuencia se realizaron a una deformación del $5 \%$, variando la frecuencia de 0.1 a $100 \mathrm{~Hz}$, se determinaron los módulos elástico y viscoso ( $G^{\prime}$ y G'), respectivamente. Lo anterior sirve de base para identificar el potencial gel de las harinas (Kong et al., 2009; Shang et al., 2021). Para determinar la naturaleza del gel se utilizó el modelo de Ross-Murphy, mediante el cual es posible determinar si un gel es fuerte o débil.

$$
\log \left(G^{\prime}\right)=\log (K)+n^{*} \log (\omega)
$$

Donde, $\mathrm{K}$ y $\mathrm{n}$ son los parámetros para determinar. El valor $(\mathrm{n})$ se considera un indicador de la naturaleza viscoelástica de los geles, si $n$ tiende a cero significa que los geles tienen tendencia a ser puramente elásticos, por el contrario, cuando n comienza a aumentar o tiende a 2, presenta un aumento en la contribución del componente viscoso. 


\section{RESULTADOS Y DISCUSION}

Este articulo pretende estudiar el efecto del tratamiento térmico sobre las estructuras secundarias de las proteínas y de la estructura interna del almidón, las cuales pueden afectar el comportamiento reológico específicamente en la estabilidad del gel, para ello, se determinó por medio de espectroscopia infrarroja los cambios de la proteína y el almidón en los diferentes geles. La figura 1 muestra la estructura del grano de quinua (a); el grano de quinua entero (b); grano de quinua pulido por 10 minutos (c); grano de quinua pulido por 40 minutos (d). Los anteriores granos fueron molidos para obtener sus respectivas harinas. De la misma manera, y con el fin de explicar mejor los resultados, se realizó la descripción detallada de las muestras, las cuales se pueden leer a continuación:

C-10-125-6\%: es una suspensión de harina del coproducto obtenido luego de la molienda abrasiva al $6 \% \mathrm{p} / \mathrm{v}$ con un tamaño de partícula menor a $125 \mu \mathrm{m}$ obtenida durante los primeros 10 minutos de la abrasión. C-40125-6\%: es una suspensión de harina del coproducto obtenido luego de la molienda abrasiva al $6 \% \mathrm{p} / \mathrm{v}$ con un tamaño de partícula menor a $125 \mu \mathrm{m}$ obtenida durante los primeros 40 minutos de la abrasión. FP-10-125$6 \%$ : es una suspensión de harina obtenida por molienda abrasiva al $6 \% \mathrm{p} / \mathrm{v}$ con un tamaño de partícula menor a $125 \mu \mathrm{m}$ obtenida durante los primeros 10 minutos de la abrasión. FP-40-125-6\%: es una suspensión de harina obtenida por molienda abrasiva al $6 \% \mathrm{p} / \mathrm{v}$ con un tamaño de partícula menor a $125 \mu \mathrm{m}$ obtenida durante los primeros 40 minutos de la abrasión. FP-10-125-T-6\%: es una suspensión de harina obtenida por molienda abrasiva al $6 \% \mathrm{p} / \mathrm{v}$ con un tamaño de partícula menor a $125 \mu \mathrm{m}$ obtenida durante los primeros 10 minutos de la abrasión, a la que posteriormente fue tratada con un proceso térmico. FP-40-125-T-6\%: es una suspensión de harina obtenida por molienda abrasiva al $6 \% \mathrm{p} / \mathrm{v}$ con un tamaño de partícula menor a $125 \mu \mathrm{m}$ obtenida durante los primeros 40 minutos de la abrasión, a la que posteriormente fue tratada con un proceso térmico. C1-125-6\%: es una suspensión de harina obtenida del grano entero al $6 \% \mathrm{p} / \mathrm{v}$ con un tamaño de partícula menor a $125 \mu \mathrm{m}$. C2-125-T-6\%: es una suspensión de harina obtenida del grano entero al $6 \% \mathrm{p} / \mathrm{v}$ con un tamaño de partícula menor a $125 \mu \mathrm{m}$, a la que posteriormente fue tratada con un proceso térmico.

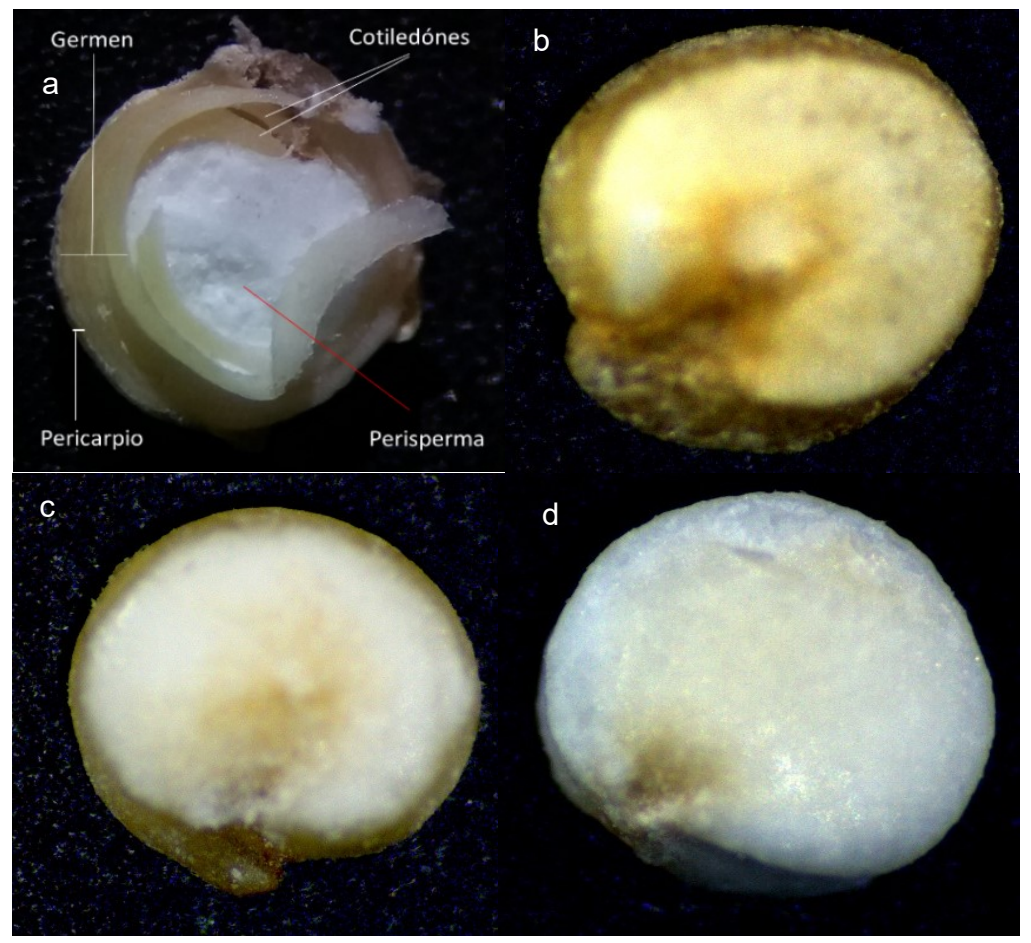

Fig. 1: Estructura del grano de quinua (a); grano de quinua entero (b); grano de quinua pulido por 10 minutos (c); grano de quinua pulido por 40 minutos (d).

\section{Espectroscopia infrarroja de los diferentes geles}

Las muestras fueron analizadas por medio de espectroscopia infrarroja, los espectros fueron normalizados y luego en el rango de la amida I, se utilizó la técnica de deconvolución para determinar la intensidad de los picos correspondientes a las estructuras secundarias de la proteína de la quinua (tabla 1) con el número de onda donde tiene la mayor intensidad de transmitancia (Yang et al., 2015). Obtenido el espectro de las estructuras secundarias de la proteína de quinua, se procedió al cálculo de las áreas relativas de los diferentes picos, para esto se determinó la línea de base más adecuada (cada valle de pico era un punto de referencia para la línea de base, esto con el fin de no alterar los espectros). Posteriormente, el espectro se aterrizó a 
una línea de base igual a cero y se procedió al cálculo de las áreas de los picos con la función integral. El método anterior resultó más conveniente para analizar los espectros en comparación al método de "fitting", ya que este método hace que la función que describe el espectro tome forma gaussiana, lo cual incrementa el error en las áreas de los picos.

En la figura 2 se observa el espectro de la harina del germen FP-40-125 y el espectro de la harina del grano pulido C-40-125, las cuales fueron obtenidas mediante molienda abrasiva durante 40 minutos. La diferencia en la forma de los espectros revela distintas estructuras proteicas, lo cual quiere decir que, la proteína del germen tiene una composición diferente en cuanto a sus estructuras secundarias con respecto a la proteína del perisperma (Roa et al., 2020). La principal diferencia que se puede observar es la desaparición de dos picos en la harina del grano pulido a los 40 minutos en las longitudes de onda correspondientes a $1641 \mathrm{~cm}^{-1}$ y $1689 \mathrm{~cm}^{-1}$ referentes a las estructuras $\beta$-sheet.
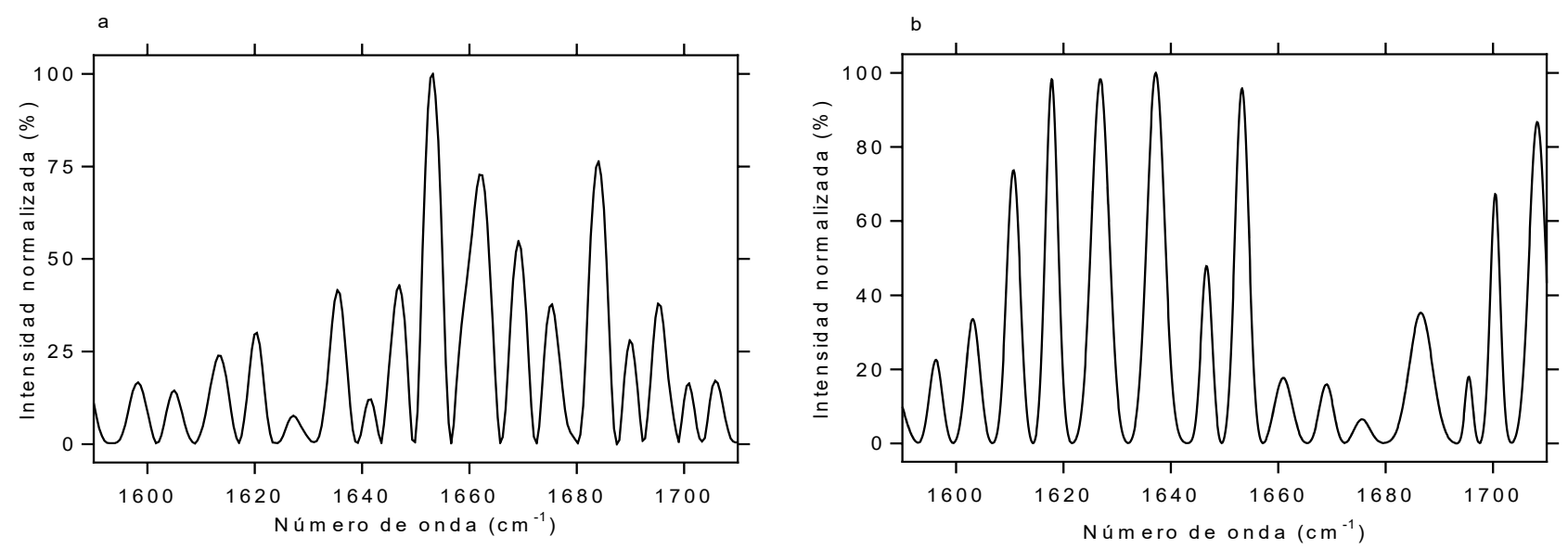

Fig. 2: Espectros FTIR (tras deconvolución) de las harinas (a) FP-40-125, (b) C-40-125.

Por otro lado, se observa en el rango del número de onda entre $1615 \mathrm{~cm}^{-1}$ a $1640 \mathrm{~cm}^{-1}$, que corresponde a las estructuras secundarias $\beta$-sheet, que la proteína del perisperma se compone principalmente de dichas estructuras, ya que el espectro de las bandas es más intenso. Por otra parte, en el rango del número de onda entre $1653 \mathrm{~cm}^{-1}$ a $1661 \mathrm{~cm}^{-1}$ se encuentran las estructuras secundarias correspondientes a $\alpha$-hélice y $3_{10-}$ hélice, respectivamente. Se observa que para la harina del germen estas bandas tienen mayor intensidad en comparación a las bandas de la harina del perisperma, este mismo comportamiento se observa para las estructuras $\beta$-turns en las bandas $1670 \mathrm{~cm}^{-1}$ a $1685 \mathrm{~cm}^{-1}$. Por lo tanto, la diferencia en las estructuras de las proteínas afecta directamente las propiedades funcionales, lo cual se describe con detalle en la sección de las propiedades reológicas.

En la figura 3 se observan los espectros correspondientes a las harinas FP-10-125 y C1-125. La diferencia en la forma de los espectros revela comportamientos diferentes en la conformación de sus proteínas. Se observa que la intensidad de las bandas en las estructuras secundarias correspondientes al grano entero es mucho mayor a comparación con las harinas de la fracción pulida a los 10 minutos, indicando que la conformación de la proteína es totalmente diferente. El espectro de la harina FP-40-125 mostró similar comportamiento con la harina FP-10-125 ya que la conformación proteica de ambas harinas es proveniente del germen. En este primer análisis se puede concluir que la molienda abrasiva es un método que permite obtener harinas con diferente composición, especialmente en la conformación de la proteína, además, se observó que la conformación de la proteína cambia dependiendo de la ubicación dentro del grano de quinua, por ejemplo, las proteínas del germen son diferentes a las proteínas presentes en el perisperma (Roa et al., 2020), y es probable que por tener diferentes proteínas en las harinas, tengan comportamientos reológicos diferentes, hipótesis que será confirmada más adelante en este trabajo de investigación.

Siguiendo con el análisis de los resultados, se estudiará el efecto hidrotérmico sobre la conformación estructural de las proteínas presentes en las harinas, para ello, es importante observar la figura 4. Esta figura presenta los espectros de las muestras FP-10-125 con y sin tratamiento hidrotérmico y las harinas FP-40-125 con y sin tratamiento hidrotérmico. Si se compara la harina FP-10-125 con FP-10-T, las estructuras afectadas por el tratamiento térmico corresponden a estructuras $\beta$-sheet $\left(1627 \mathrm{~cm}^{-1}\right.$ y $\left.1635 \mathrm{~cm}^{-1}\right)$ y $\beta$-turns $\left(1661 \mathrm{~cm}^{-1}\right)$. Sin embargo, el efecto más notorio es sobre las harinas FP-40-125 y FP-40-125-T, donde el tratamiento hidrotérmico es capaz de cambiar todas las estructuras $\beta$-sheet $\left(1619 \mathrm{~cm}^{-1}, 1627 \mathrm{~cm}^{-1}, 1635 \mathrm{~cm}^{-1}\right)$ y $\beta$-turns $\left(1661 \mathrm{~cm}^{-1}, 1669 \mathrm{~cm}^{-1}\right)$, así como, las estructuras $\alpha$-hélice $\left(1653 \mathrm{~cm}^{-1}\right)$, es probable que el efecto térmico afecte más estructuras secundarias en la harina FP-40, ya que esta harina tiene proteínas del germen, aleurona, así como, proteínas ubicadas en las primeras capas del perisperma (Hemalatha et al., 2016; Mamani et al., 2017). 

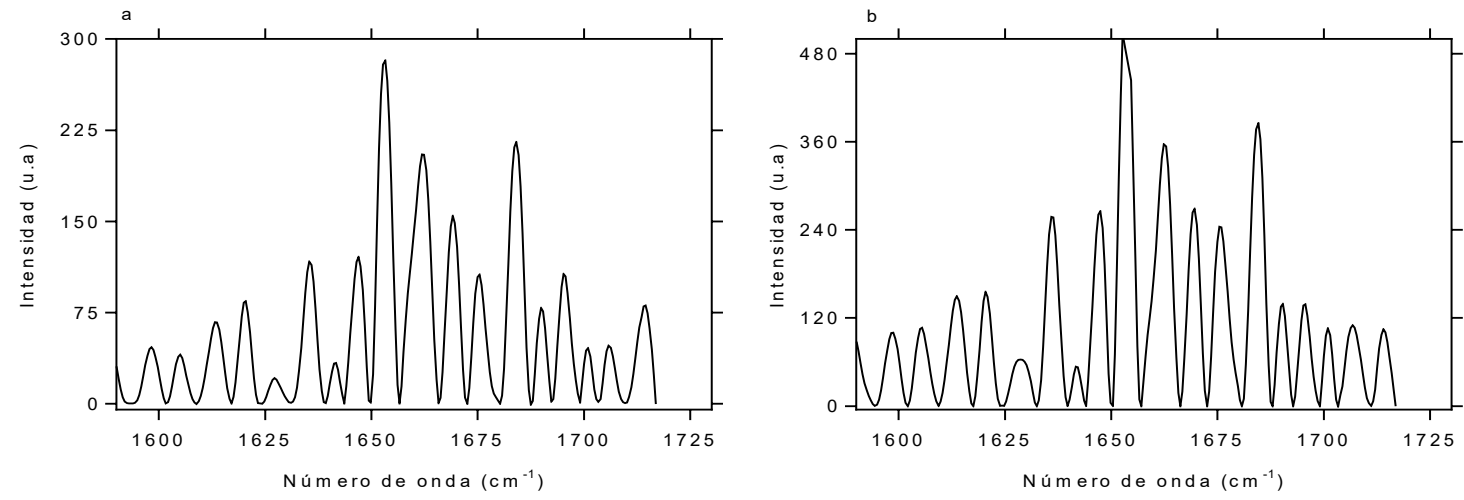

Fig. 3: Espectros FTIR (tras deconvolución) de las harinas (a) FP-10-125, (b) C1-125.
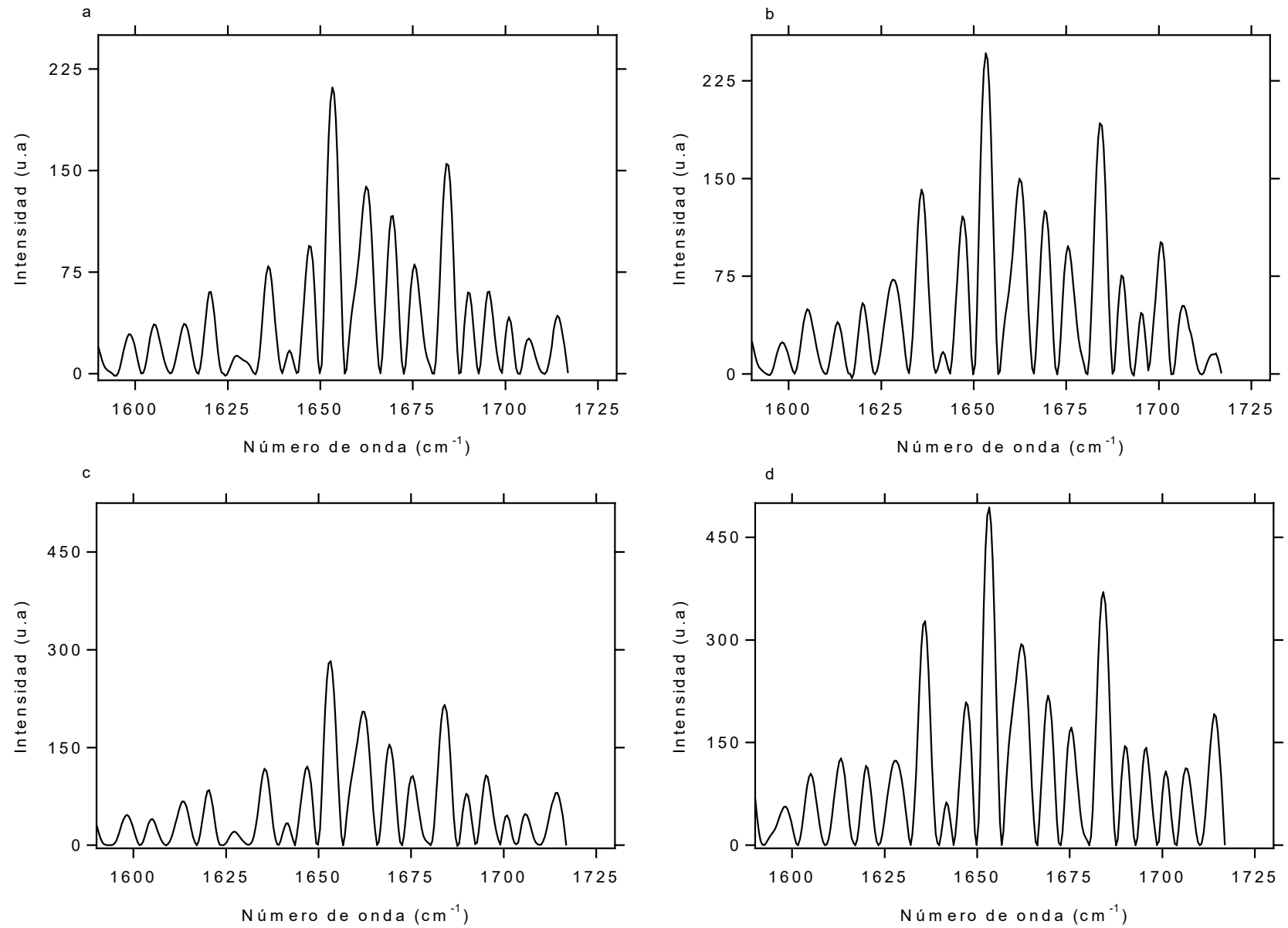

Fig. 4: Espectros FTIR (tras deconvolución) de las harinas (a) FP-10-125 (b) FP-10-125-T (c) FP-40-125, (d) FP-40-125-T.

Con el fin de resumir los resultados se identificaron las bandas características de cada estructura secundaria de la proteína de quinua, se realizó el análisis estadístico para determinar diferencias significativas en las áreas de los picos mediante ANOVA y comparación múltiples t-test con un nivel de confianza del 95\%. Los cambios más significativos fueron en las siguientes bandas: $\beta$-sheet $\left(1627 \mathrm{~cm}^{-1}\right), \beta$-turns $\left(1661 \mathrm{~cm}^{-1}\right), \alpha$-helix $\left(1653 \mathrm{~cm}^{-1}\right)$ y Antiparallel $\beta$-sheet $\left(1613 \mathrm{~cm}^{-1}\right)$. La figura 5 muestra en resumen el procesamiento hidrotérmico de las harinas y su efecto en las estructuras secundarias de las proteínas presentes en las harinas. Se observa que el almidón tiene picos en el rango de $800 \mathrm{~cm}^{-1}-1200 \mathrm{~cm}^{-1}$ que son típicos de los polisacáridos. Los picos característicos de las muestras que contienen almidón generalmente se ubican en el número de onda de 1010 $\mathrm{cm}^{-1}, 1080 \mathrm{~cm}^{-1}$ y $1150 \mathrm{~cm}^{-1}$, que se asignan a los estiramientos $\mathrm{C}-\mathrm{O}$ y $\mathrm{C}-\mathrm{C}$ en las moléculas de los polisacáridos, principalmente a las vibraciones de los enlaces glucosídicos $\mathrm{C}-\mathrm{O}-\mathrm{C}$ y $\mathrm{C}-\mathrm{O}-\mathrm{H}$ (Teng et al., 2021). Los espectros que son sometidos al proceso de deconvolución por medio de algunas de las funciones como la Fourier, Gaussianas y Lorenzianas, se emplean para estudiar el orden estructural del granulo de almidón, esto es muy importante, ya que el orden estructural se vincula a la funcionalidad del almidón. 


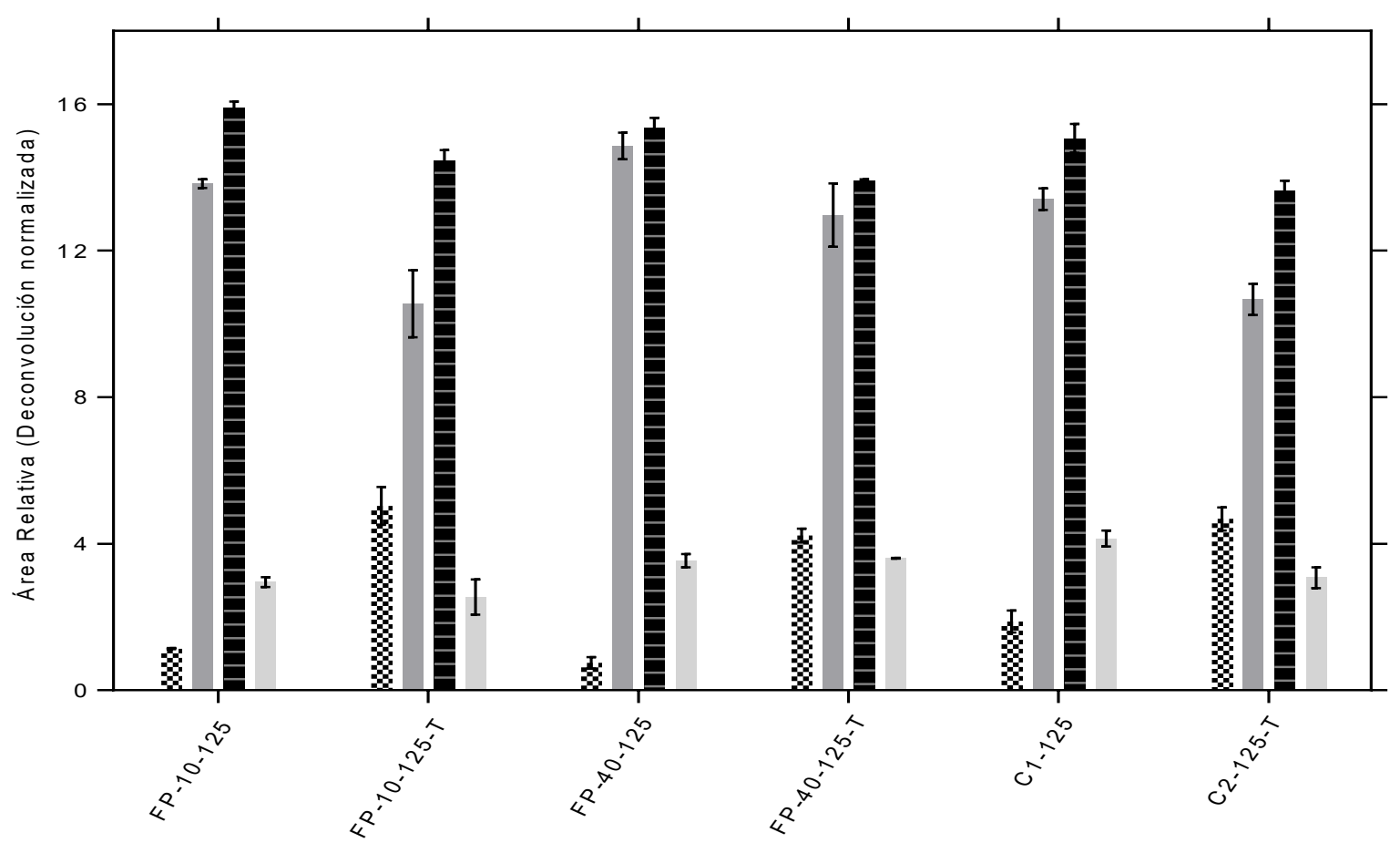

Fig. 5: Comparación entre las intensidades de las estructuras secundarias en las muestras sometidas a tratamientos hidrotérmico. En orden de izquierda a derecha, las barras son: $\beta$-sheet $\left(1627 \mathrm{~cm}^{-1}\right), \beta$-turns $\left(1661 \mathrm{~cm}^{-1}\right), \alpha$-helix $\left(1653 \mathrm{~cm}^{-}\right.$ $\left.{ }^{1}\right)$, Antiparallel $\beta$-sheet $\left(1613 \mathrm{~cm}^{-1}\right)$.

Una vez, entendido lo anterior se continua con el análisis de los resultados del efecto térmico sobre la conformación estructural de los carbohidratos especialmente en la organización interna de los gránulos de almidón presentes en las harinas, para ello, es importante observar la figura 6 . Se observa los espectros de las muestras con y sin tratamiento térmico, además, se identifican 5 picos $\left(P_{1}, P_{2}, P_{3}, P_{4}, P_{5}\right)$ los cuales están relacionadas a las intensidades de las longitudes de onda $1012 \mathrm{~cm}^{-1}, 1040 \mathrm{~cm}^{-1}, 1071 \mathrm{~cm}^{-1}, 1096 \mathrm{~cm}^{-1}, 1137$ $\mathrm{cm}^{-1}$, respectivamente. La relación $\mathrm{P}_{1} / \mathrm{P}_{2}$ ha sido descrita por varios autores (Pardo et al., 2013; Wang et al., 2020) como un índice adecuado de la relación amorfo/cristalino del almidón; lo anterior quiere decir que, si la relación aumenta, es porque el almidón es más amorfo. Las muestras que fueron sometidas a un tratamiento térmico (líneas continuas) mostraron una relación $\mathrm{P}_{1} / \mathrm{P}_{2}$ mayor a las muestras que no fueron sometidas al tratamiento (líneas punteadas) (figura 6). Se observa que el tratamiento térmico incremento el pico $P_{1}$ y simultáneamente disminuyo el pico $\mathrm{P}_{2}$, esto quiere decir que, el almidón sufrió una modificación en su estructura interna, donde las estructuras ordenadas (cristalinas) disminuyen y se incrementan las estructuras desordenadas (amorfas) (Mir et al., 2021).

Es importante destacar, que la relación de intensidades $\mathrm{P}_{1} / \mathrm{P}_{2}$ en las muestras FP-10 y FP-40 son iguales, pero mayores a la relación $\mathrm{P}_{1} / \mathrm{P}_{2}$ en la harina entera; lo que indica que el almidón presente en las muestras FP-10 y FP-40 es más amorfo, mientras el almidón de la harina entera es más cristalino. Surge lo siguiente ¿Por qué el almidón de las harinas tienen diferente organización estructural si son del mismo grano de la quinua?, la respuesta puede ser abordada desde el proceso que se empleó para obtener las harinas; como en la molienda abrasiva se realiza un pulimiento del grano por capas, en la cual se van eliminando las partes exteriores, que en el caso de las muestras FP-10 y FP-40 sería las primeras capas del perisperma almidonoso, mientras que en la harina entera el almidón proviene de todo el perisperma (exterior e interior) (figura 1). Por lo tanto, se podría decir que el almidón ubicado en la superficie del perisperma es más amorfo que el almidón ubicado en el interior del perisperma, esto podría explicar por qué el tratamiento térmico afecta más la estructura del almidón en las muestras FP-10 y FP-40, donde se evidencia que a relación $\mathrm{P}_{1} / \mathrm{P}_{2}$ es mayor en comparación a la de la harina entera.

La relación de intensidad de las bandas a $1137 \mathrm{~cm}^{-1}$ y $1071 \mathrm{~cm}^{-1}$ (1137/1071) se empleó como un índice del contenido de almidón, se observó un incremento de esta relación en la harina entera, mientras que, en las harinas FP-10 y FP-40 se observó diferencia significativa en lo relacionado a $P_{5} / P_{3}$. Por otra parte, el tratamiento térmico no afectó significativamente la relación $\mathrm{P}_{5} / \mathrm{P}_{3}$. Finalmente, el pico $\mathrm{P}_{4}$ que corresponde a la banda $1096 \mathrm{~cm}^{-1}$, puede ser empleado para distinguir entre las muestras sometidas y no procesadas térmicamente, intensidades de $\mathrm{P}_{4}$ mayores a 0.4 corresponden a harinas procesadas térmicamente. 


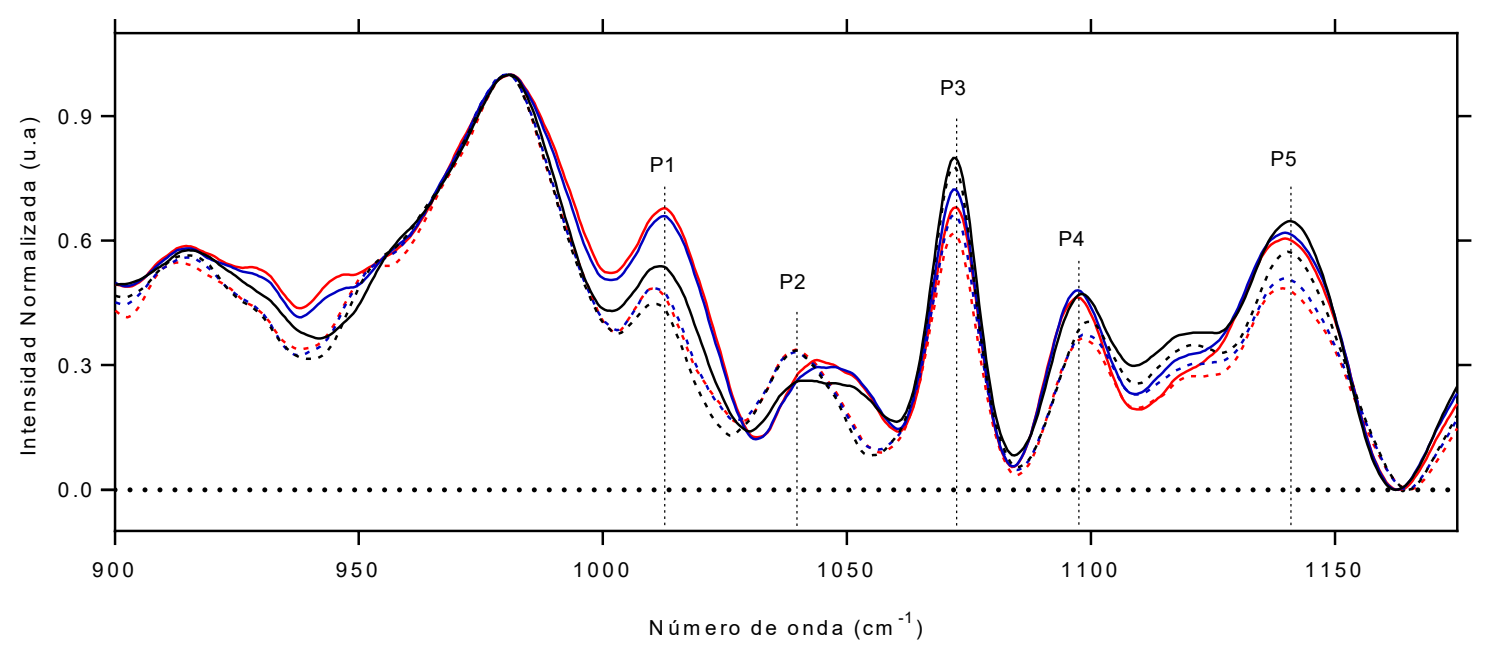

Fig. 6: Organización molecular del almidón en las diferentes harinas: - C2-125-T, - - - C1-125-6, - FP-40-125-T, - - - FP-40-125, - FP-10-125-T, - - - FP-10-125

\section{Comportamiento viscoelástico}

El módulo G' es una medida de la energía que es almacenada en el material y es recuperable, lo cual es propio del comportamiento sólido de una muestra. El módulo G" es una medida de la energía que se disipa y que es propia del comportamiento líquido de una muestra (López-Monterrubio et al., 2020). Con el fin de profundizar la caracterización y el efecto del procesamiento térmico sobre las características reológicas de las diferentes dispersiones, se determinó el comportamiento de los módulos $G^{\prime}$ y $G^{\text {" }}$ con el incremento de la temperatura, en el aislado proteico, y en las diferentes harinas.

La figura 7 muestra la evolución de los módulos $G^{\prime}$ y G", se observa poca variación de los módulos durante el calentamiento hasta los $80^{\circ} \mathrm{C}$, sin embargo, el módulo $\mathrm{G}^{\prime}$ es mayor que el G'. A partir de los $80^{\circ} \mathrm{C}$ se registra un salto en el módulo $G^{\prime}$ (cuadrado rojo) y simultáneamente hay una fuerte caída del ángulo de fase (circulo negro) esta caída está asociada al incremento del valor $G^{\prime}$, dicho incremento, se da por la desnaturalización de la proteína y la consecuente formación del gel, esto confirma que a esa temperatura la proteína de la harina es desnaturalizada provocando un aumento en la viscosidad y este aumento se refleja en el incremento del módulo G'.

El módulo G" es ligeramente mayor a la modulo G’, lo que indica un comportamiento típico de la dispersión liquida hasta los $45^{\circ} \mathrm{C}$, luego hay un entrecruzamiento de los módulos, lo que muestra la formación de algún gel, dado que esto se da a una temperatura de $45^{\circ} \mathrm{C}$ no es posible asociar esto a la desnaturalización de la proteína, este entrecruzamiento puede estar asociado al inicio de la hidratación de los gránulos de almidón, se observa que el módulo G' sigue incrementándose gracias a la gelatinización del almidón que puede estar ocurriendo a temperaturas cercanas a los $60^{\circ} \mathrm{C}$ y menores a $80^{\circ} \mathrm{C}$, si se sigue calentando la dispersión, el módulo G’ sigue incrementándose ya que se inicia el proceso de desnaturalización de la proteína a temperaturas mayores $\left(85^{\circ} \mathrm{C}\right)$, en este caso no se observa el entrecruzamiento, ya que el gel ya se formó y el comportamiento elástico ya se había evidenciado.

La figura 7a,c muestra el comportamiento de las dispersiones FP-10 y FP-40 respectivamente, en cuanto al comportamiento del módulo elástico $G^{\prime}$ es similar en las dos dispersiones, se observa un incremento después de los $60^{\circ} \mathrm{C}$ que es atribuido a la gelatinización del almidón presente en el muestra, no se observa entrecruzamiento de los módulos en todo el barrido de temperatura indicando la características elásticas de la dispersión y gel formado durante la gelatinización del almidón y posteriormente la desnaturalización de la proteína presente. Es de destacar que la dispersión FP-10 es más inestable que la dispersión FP-40, esto se observa por la caída del ángulo de fase en la dispersión FP-10, el ángulo de fase cae desde $70^{\circ}$ hasta $20^{\circ}$, lo que muestra la dependencia de esta dispersión al calentamiento. Sin embargo, en la dispersión FP-40, la disminución del ángulo de fase se da desde los $35^{\circ}$ hasta los $10^{\circ}$ una caída mucho menor, indicando un comportamiento elástico más estable. Posiblemente, esta estabilidad es brindada por el mayor contenido de proteína, esta proteína puede formar redes con el agua y otros compuestos, y de esta manera estabilizar la dispersión (Yamul, 2008). Las figuras $7 \mathrm{~b}, \mathrm{~d}$ muestra el comportamiento de las harinas con tratamiento térmico; en todo el rango de temperatura el módulo de perdida $\left(G^{\prime \prime}\right)$ es mayor a modulo elástico ( $\left.G^{\prime}\right)$ esto evidencia el comportamiento liquido de la muestra, además, la muestra no es afectada en el calentamiento brindándole estabilidad térmica, lo cual puede ser observado en la oscilación del ángulo de fase entre $40^{\circ}$ y $60^{\circ}$ en este rango no hay casi diferencia entre los módulos y está relacionado a la estabilidad reológica. 

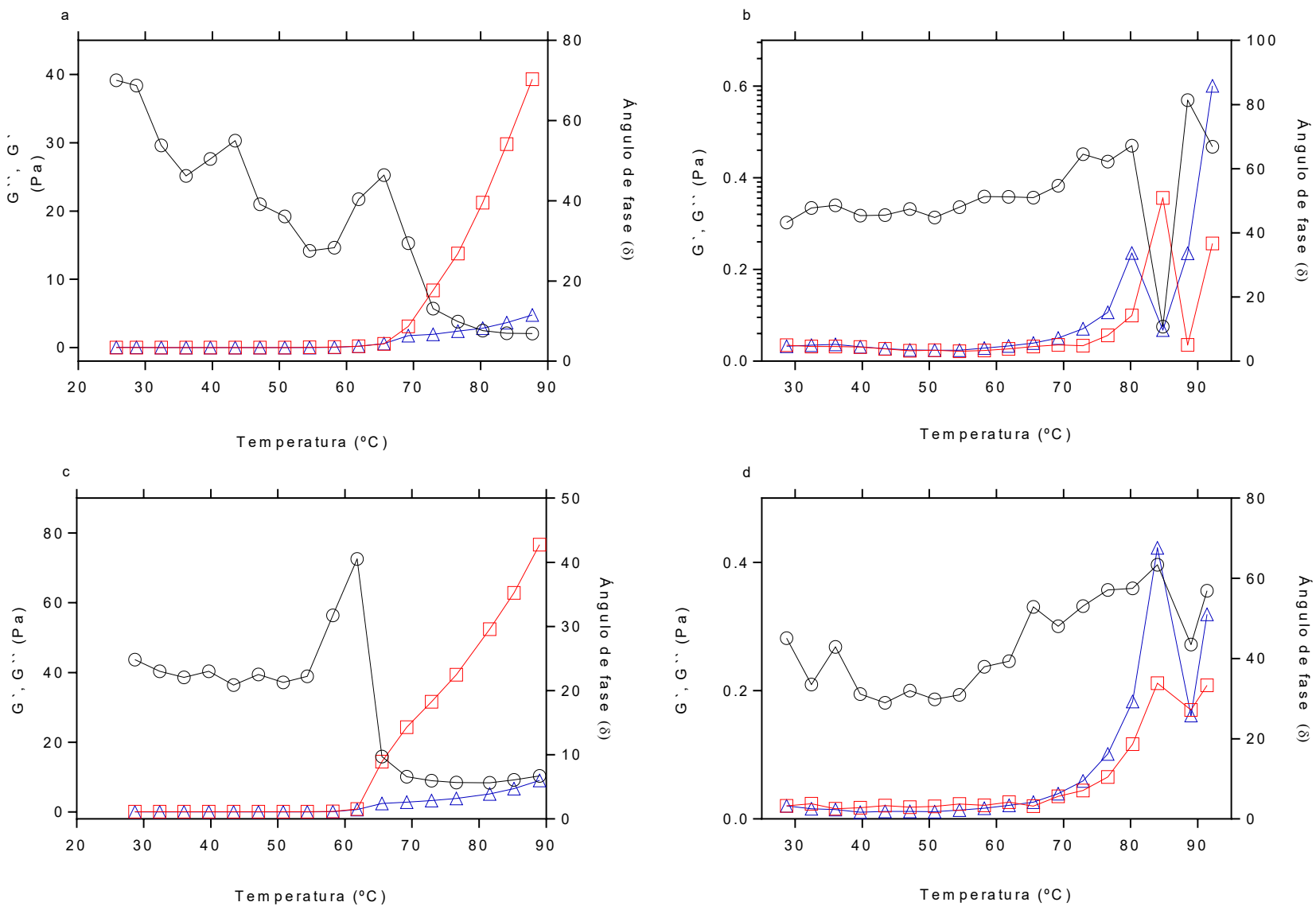

Fig. 7: Comportamiento reológico de las dispersiones FP-10-125-6\% (a), FP-10-125-T-6\% (b), FP-40-125-6\% (c), FP-40$125-\mathrm{T}-6 \%$ (d). Los símbolos representan: $\bigcirc$ Ángulo de fase; $\square G^{\prime} ; \triangle G^{\prime \prime}$.

\section{Estabilidad del gel}

La influencia de la frecuencia en los módulos dinámicos G' y G" de los geles se observan en la figura 8. Según Steffe (1996) a partir de los resultados del barrido de frecuencia podemos distinguir diferentes tipos de dispersión y geles. Se encontró a partir de los espectros mecánicos de los geles evaluados dentro del rango de frecuencia de 0.1 a $100 \mathrm{~Hz}$ que en todas las muestras el módulo elástico G' excede el módulo G". Los geles obtenidos de las muestras de las fracciones pulidas y grano entero con o sin tratamiento térmico no presentan ningún punto de cruce entre los módulos viscoelásticos, esto es favorable en lo que respecta a la estabilidad del gel. Para el barrido de frecuencia se utilizó el modelo de Ross-Murphy, el cual ajusto satisfactoriamente $\left(R^{2}>0.97\right)$, mediante este modelo se logró determinar el índice de estabilidad de gel $(n)$.

La figura 8a,b muestra el comportamiento del gel a partir de las dispersiones FP-10 y FP-10-T respectivamente, en cuanto al módulo de almacenamiento $\left(G^{\prime}\right)$ y el módulo de perdida ( $\left.G^{\prime \prime}\right)$ se observa un incremento de los módulos y su dependencia a la frecuencia para las dos muestras, sin embargo, la muestra sin tratamiento térmico (FP-10) logro una diferencia en el valor del $\mathrm{G}^{\text {' }}$ desde $2723 \mathrm{~Pa}$ hasta $12162 \mathrm{~Pa}$; mientras que la muestra sometida a tratamiento térmico (FP-10-T) presentó un incremento menor (1122 Pa a $4750 \mathrm{~Pa})$, es probable que los geles FP-10 sean más firmes por los altos valores de G', esto indica que el tratamiento térmico genera geles con menos firmeza, siempre y cuando se use muestra FP-10. Por otra parte, el índice de estabilidad de gel $(n)$ no mostro diferencias significativas $(p<0.05)$. Esto quiere decir que los geles obtenidos a partir de las muestras FP-10 con y sin tratamiento térmico podrían comportase similarmente en cuanto a su estabilidad.

La figura 8c,d muestra el comportamiento del gel formado de las dispersiones FP-40 y FP-40-T respectivamente, en cuanto al módulo de almacenamiento $\left(G^{\prime}\right)$ y el módulo de perdida ( $\left.G^{\prime \prime}\right)$ se observa que las muestras presentan un incremento de sus módulos dependientes de la frecuencia (2377 Pa hasta 11885 $\mathrm{Pa}$ ) y (214 Pa hasta $708 \mathrm{~Pa}$ ), respectivamente; este comportamiento fue similar a las observadas en las muestras FP-10. Sin embargo, si se observaron diferencias entre los índices ( $\mathrm{n}$ ), por ejemplo, para la muestra FP-40-T se determinó un índice $(n=0.1621)$, mientras que para la muestra FP-40 un índice $(n=0.2174)$. Teniendo en cuenta estudios anteriores de Roa et al. (2017) los cuales establecieron que los geles de tipo fuerte presentan un índice ( $\mathrm{n}$ ) con tendencia a 0 y los geles de débil firmeza tienden a un índice $n=0.3$ en 
geles obtenidos a partir de harina de amaranto, siendo este un pseudocereal como la quinua. Por lo tanto, podemos decir que los geles a partir de la muestra FP-40 son más firmes, pero menos estables en el tiempo, mientras que los geles FP-40-T son menos firmes, pero con más estabilidad en el tiempo (menos dependientes de la frecuencia), en otras palabras, el efecto del tratamiento térmico provoca estabilidad en este tipo de muestras. La figura 8e,f muestra el comportamiento del gel formado de las dispersiones de la harina del grano entero y harina del grano entero con tratamiento térmico, respectivamente. Estas muestras se comportaron similarmente a las descritas en las muestras FP-40, donde el tratamiento térmico proporciona mejor estabilidad en los geles.
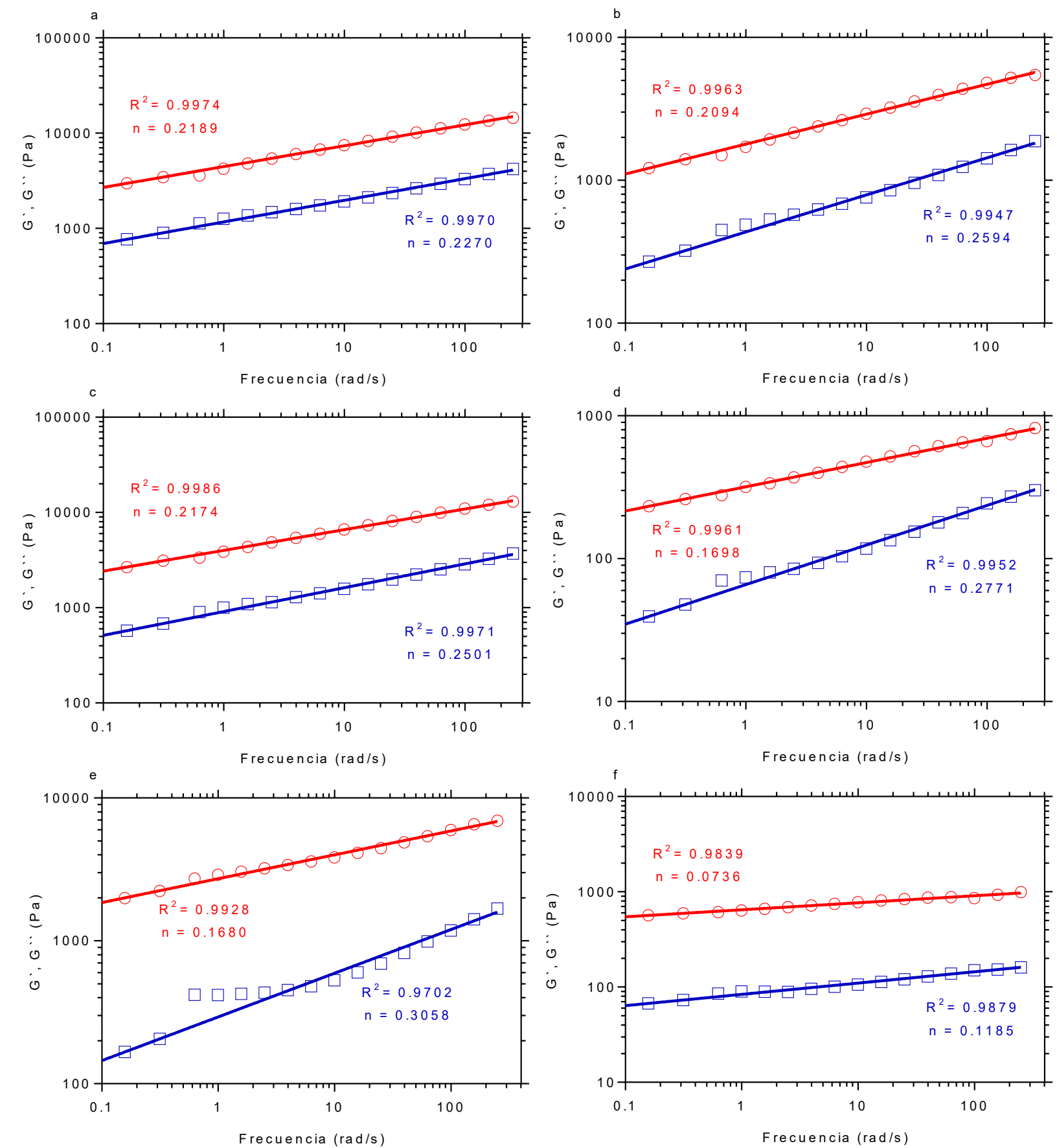

Fig. 8: Barrido de frecuencia de los geles formados a partir de harina FP-10-125-6\% (a), FP-10-125-T-6\% (b), FP-40-1256\% (c), FP-40-125-T-6\% (d), C1-125-6\% (e), C2-125-T-6\% (f). Los símbolos representan: $\theta$ G'; $\square$ G".

Las propiedades reológicas de los geles elaborados a partir de las muestras FP-10 y FP-40 fueron similares a pesar de su diferente composición, siendo la FP-10 más fibrosa y FP-40 más proteica, sin embargo, la cantidad de almidón fue similar en las dos muestras. La diferencia en la composición de las figuras 7a,c y las figura $7 \mathrm{~b}, \mathrm{~d}$ es la misma, sin embargo, el índice $\mathrm{n}$ para la muestra $8 \mathrm{a}, \mathrm{c}$ es similar, lo cual indica que la composición de la muestra no influye en la estabilidad del gel, mientras que para las muestras $8 \mathrm{~b}, \mathrm{~d}$ el valor del índice $\mathrm{n}$ se ve influenciado por efecto del tratamiento térmico, el cual modifica la funcionalidad de la 
composición por el hecho de que hay un mayor contenido de proteína en la muestra FP-40 en comparación con FP-10 (Solaesa et al., 2020). El tratamiento térmico produce la desnaturalización de las proteínas lo que probablemente propicia formación de uniones entre las cadenas de proteína y contribuyen a formar una estructura más dura y fuerte $(n=0,1698)$ (Roa et al., 2017). Las propiedades funcionales y reológicas de las harinas dependen en gran medida de la cantidad de almidón y la relación amilosa/amilopectina (SolarteMontúfar et al., 2019). El índice de la figura $8 b, d$ es mayor $(n=0.2094$ y $n=0.1698)$, respectivamente al de la figura $8 f(n=0.0736)$ lo cual indica que las fracciones pulidas presentan una estructura más débil que se rompe más fácil bajo estrés, atribuible a su baja cantidad de almidón y a su alto contenido de proteínas, contrario al grano entero con tratamiento térmico que presenta un gel más fuerte por su alto contenido de almidón que influye directamente en su estabilidad durante el almacenamiento (Ayseli et al., 2020).

\section{CONCLUSIONES}

La molienda abrasiva resulto ser una operación eficiente para lograr obtener dos tipos de harinas a partir del grano de quinua, una harina con un contenido de proteína y grasa elevado, que se denominó harina del germen y una harina con alto contenido de almidón descrita como harina del perisperma. Cada una de las harinas mostro propiedades tecnofuncionales diferentes, esto se debe principalmente al tipo de relación cristalino/amorfo que existe en el almidón. Sin embargo, como todo almidón, sus geles tienden a retrogradar y a mostrar inestabilidad en el tiempo. Este problema fue solucionado con un pretratamiento térmico que se les aplican a las harinas, por medio de este tratamiento, se logró obtener geles no dependientes de la frecuencia, lo que indica una buena estabilidad. Por lo tanto, la combinación de la molienda abrasiva, seguida del tratamiento térmico, permite obtener harinas con propiedades tecnofuncioanles diferentes a las harinas comunes.

\section{REFERENCIAS}

Ahmed, J., Thomas, L., Arfat, Y.A., y Joseph, A., Rheological, structural and functional properties of high-pressure treated quinoa starch in dispersions, https://doi.org/10.1016/j.carbpol.2018.05.081, Carbohydrate Polymers, 197, 649657 (2018)

Ayseli, M.T., Yilmaz, M.T., y otros cuatro autores, Physicochemical, rheological, molecular, thermal and sensory evaluation of newly developed complementary infant (6-24 months old) foods prepared with quinoa (Chenopodium quinoa Willd.) flour, https://doi.org/10.1016/j.foodchem.2020.126208, Food Chemistry, 315, 1-13 (2020)

Ballester-Sánchez, J., Fernández-Espinar, M., y Haros, C., Isolation of red quinoa fibre by wet and dry milling and application as a potential functional bakery ingredient, https://doi.org/10.1016/j.foodhyd.2019.105513, Food Hydrocolloids, 69, 1-9 (2020)

Bolade, M.K., Usman, M.A., y otros tres autores, Influence of hydrothermal treatment of maize grains on the quality and acceptability of Tuwon masara (traditional maize gel), https://doi.org/10.1016/s0308-8146(02)00223-6, Food Chemistry, 79(4), 479-483 (2002)

Calzetta, A.N., Tolaba, M.P., y Suárez, C., Effects of steeping conditions on wet-milling attributes of amaranth, https://doi.org/10.1111/j.1365-2621.2006.01395.x, International Journal of Food Science and Technology, 41(s2), 70-76 (2006)

Dussán-Sarria, S., De la Cruz-Noguera, R., y Godoy, S. P., Estudio del Perfil de Aminoácidos y Análisis Proximal de Pastas Secas Extruidas a Base de Harina de Quinua y Harina de Chontaduro, https://doi.org/10.4067/s071807642019000600093, Información Tecnológica, 30(6), 93-100 (2019)

Ghumman, A., Mudgal, S., y otros cuatro autores, Physicochemical, functional and structural characteristics of grains, flour and protein isolates of Indian quinoa lines, https://doi.org/10.1016/j.foodres.2020.109982, Food Research International, 140, 1-9 (2021)

Hemalatha, P., Bomzan, D., Sathyendra Rao, B., y Sreerama, Y., Distribution of phenolic antioxidants in whole and milled fractions of quinoa and their inhibitory effects on $\alpha$-amylase and $\alpha$-glucosidase activities, https://doi.org/10.1016/j.foodchem.2015.12.025, Food Chemistry, 199, 330-338 (2016)

Kong, X., Kasapis, S., Bao, J., y Corke, H., Effect of gamma irradiation on the thermal and rheological properties of grain amaranth starch, https://doi.org/10.1016/j.radphyschem.2009.07.019, Radiation Physics and Chemistry, 78(11), 954-960 (2009)

Le, L., Gong, X., y otros diez autores, Quinoa sprouts as potential vegetable source: Nutrient composition and functional contents of different quinoa sprout varieties, https://doi.org/10.1016/j.foodchem.2021.129752, Food Chemistry, 357, 129752 (2021)

López-Monterrubio, D., Lobato-Calleros, C., Alvarez-Ramirez, J., y Vernon-Carter, E., Huauzontle (Chenopodium nuttalliae Saff.) protein: Composition, structure, physicochemical and functional properties,

https://doi.org/10.1016/j.foodhyd.2020.106043, Food Hydrocolloids, 108, 106043 (2020) 
Mamani, D., Gutierrez, M., Serrudo, J., y Gonzales, E., Parámetros de calidad de harinas de Amaranthus caudatus Linnaeus (amaranto), Chenopodium quinoa Willd (quinua), Chenopodium pallidicaule Aellen (kañahua), Lupinus mutabilis Sweet (tarwi), Con-Ciencia, 15(1), 27-38 (2017)

Mir, N.A., Riar, C.S., y Singh, S., Improvement in the functional properties of quinoa (Chenopodium quinoa) protein isolates after the application of controlled heat-treatment: Effect on structural properties, https://doi.org/10.1016/j.foostr.2021.100189, Food Structure, 28, 100189 (2021)

Morillo, A. C., Castro, M. A., y Morillo, Y., Caracterización de la diversidad genética de una colección de Quinua (Chenopodium quinoa Willd), https://doi.org/10.18684/bsaa(15)49-56, Biotecnología en el Sector Agropecuario y Agroindustrial, 15(2), 49-56 (2017)

Ngamnikom, P., y Songsermpong, S., The effects of freeze, dry, and wet grinding processes on rice flour properties and their energy consumption, https://doi.org/10.1016/j.jfoodeng.2011.02.001, Journal of Food Engineering, 104(4), 632-638 (2011)

Pardo, O., Castañeda, J., y Ortiz, C., Caracterización estructural y térmica de almidones provenientes de diferentes variedades de papa, Acta Agronómica, 62(4), 289-295 (2013)

Roa, D., Ortiz, V., y Tolaba, M., Comportamiento reológico de la fracción proteica del grano de amaranto extraída mediante molienda húmedo-ácida, Biotecnología en el Sector Agropecuario y Agroindustrial, 15(1), 123-130 (2017)

Roa-Acosta, D.F., Bravo-Gómez, J.E., y otros tres autores, Hyper-protein quinoa flour (Chenopodium quinoa Wild) Monitoring and study of structural and rheological properties, https://doi.org/10.1016/j.Iwt.2019.108952, LWT, 121, 108952 (2020)

Rodríguez, M. J., Prieto, J.M., Cruz, V., y Calvo, P., Nutritional characterization of six quinoa (Chenopodium quinoa Willd) varieties cultivated in Southern Europe, https://doi.org/10.1016/j.jfca.2021.103876, Journal of Food Composition and Analysis, 99, 103876 (2021)

Shang, J., LI, L., y otros cinco autores, Relationships of flour characteristics with Isolated Starch Properties in Different Chinese Wheat Varieties, https://doi.org/10.1016/j.jcs.2021.103210, Journal of Cereal Science, 99, 1-9 (2021)

Solaesa, Á.G., Villanueva, M., Vela, A.J., y Ronda, F., Protein and lipid enrichment of quinoa (cv.Titicaca) by dry fractionation. Techno-functional, thermal and rheological properties of milling fractions, https://doi.org/10.1016/j.foodhyd.2020.105770, Food Hydrocolloids, 105, 105770 (2020)

Solarte-Montúfar, J., Díaz-Murangal, A., Osorio-Mora, O., y Mejía-España, D., Propiedades Reológicas y Funcionales del Almidón. Procedente de Tres Variedades de Papa Criolla, https://doi.org/10.4067/s0718-07642019000600035, Información Tecnológica, 30(6), 35-44 (2019)

Steffe, J., Rheological methods in foods process engineering, $2^{a}$ Ed., 418, University Press, Amsterdam, Países Bajos (1996)

Teng, C., Qin, P., y otros cinco autores, Structural characterization and antioxidant activity of alkali-extracted polysaccharides from quinoa, https://doi.org/10.1016/j.foodhyd.2020.106392, Food Hydrocolloids, 113, 1-10 (2021)

Turkut, G.M., Cakmak, H., Kumcuoglu, S., y Tavman, S., Effect of quinoa flour on gluten-free bread batter rheology and bread quality, https://doi.org/10.1016/j.jcs.2016.03.005, Journal of Cereal Science, 69, 174-181 (2016)

Wang, X., Zhao, R., y Yuan, W., Composition and secondary structure of proteins isolated from six different quinoa varieties from China, https://doi.org/10.1016/j.jcs.2020.103036, Journal of Cereal Science, 95, 103036 (2020)

Xing, B., Teng, C., y otros siete autores, Effect of germination treatment on the structural and physicochemical properties of quinoa starch, https://doi.org/10.1016/j.foodhyd.2021.106604, Food Hydrocolloids, 115, 106604 (2021)

Yamul, D., "Propiedades de geles de concentrado de proteínas de lactosuero, miel y harina", Trabajo de grado Doctor, Facultad de Ciencias Exactas, Universidad Nacional de La Plata, Centro de Investigación y Desarrollo en Criotecnología de Alimentos (2008)

Yang, H., Yang, S., y otros tres autores, Obtaining information about protein secondary structures in aqueous solution using Fourier transform IR spectroscopy, https://doi.org/10.1038/nprot.2015.024, Nature Protocols, 10(3), 382-396 (2015)

Zhu, F., y Wang, Y. J., Rheological and thermal properties of rice starch and rutin mixtures, https://doi.org/10.1016/j.foodres.2012.09.031, Food Research International, 49(2), 757-762 (2012) 